\title{
Clinical efficacy and molecular biomarkers in a phase II study of tucidinostat plus R-CHOP in elderly patients with newly diagnosed diffuse large B-cell lymphoma
}

Mu-Chen Zhang ${ }^{1 \dagger}$, Ying Fang ${ }^{1 \dagger}$, Li Wang ${ }^{1,2+}$, Shu Cheng ${ }^{1}$, Di Fu' ${ }^{1}$, Yang He ${ }^{1}$, Yan Zhao ${ }^{1}$, Chao-Fu Wang ${ }^{3}$, Xu-Feng Jiang ${ }^{4}$, Qi Song ${ }^{5}$, Peng-Peng $X^{1{ }^{*}}$ and Wei-Li Zhao ${ }^{1,2^{*}}$

\begin{abstract}
Background: Elderly patients with diffuse large B-cell lymphoma (DLBCL) present with poor clinical outcome and intolerance to intensive chemotherapy. Histone deacetylase inhibitors (HDACls) show anti-lymphoma activities and can be applied to treat DLBCL. This study aimed to evaluate efficacy and safety of oral HDACI tucidinostat (formerly known as chidamide) plus R-CHOP (CR-CHOP) in elderly patients with newly diagnosed DLBCL (International Prognostic Index $\geq 2$ ).
\end{abstract}

Results: Among 49 patients, the complete response rate was $86 \%$, with overall response rate achieving $94 \%$. The 2-year progression survival (PFS) and overall survival (OS) rates were 68\% (95\% Cl 52-79) and 83\% (95\% Cl 68-91). Comparing with historical control (NCT01852435), the 2-year PFS and OS rates of double-expressor lymphoma phenotype (DEL) were improved, and negative prognostic effect of histone acetyltransferases CREBBP/EP300 mutations was also mitigated by CR-CHOP. Grade 3-4 neutropenia was reported in 171, grade 3-4 thrombocytopenia in 27, and grade 3 anemia in 11 of 283 cycles. No grade 4 non-hematological adverse event was reported.

Conclusion: $\mathrm{CR}-\mathrm{CHOP}$ is effective and safe in elderly patients with newly diagnosed DLBCL. Relevance of DEL phenotype and molecular biomarkers on CR-CHOP response warrants further investigation in DLBCL.

Trial registration ClinicalTrial.gov, NCT02753647. Registered on April 28, 2016.

Keywords: Diffuse large B-cell lymphoma, Double expressor lymphoma, Histone deacetylase inhibitor, Tucidinostat, CREBBP/EP300

*Correspondence: pengpeng_xu@126.com; zhao.weili@yahoo.com ${ }^{\dagger} \mathrm{Mu}-$ Chen Zhang, Ying Fang and Li Wang have contributed equally to this manuscript

1 Shanghai Institute of Hematology, State Key Laboratory of Medical Genomics, National Research Center for Translational Medicine At

Shanghai, Ruijin Hospital Affiliated To Shanghai Jiao Tong University School of Medicine, Shanghai, China

Full list of author information is available at the end of the article

\begin{abstract}
Background
Diffuse large B-cell lymphoma (DLBCL) represents the most common subtype of non-Hodgkin's lymphoma and is heterogeneous in clinical, immunophenotypic, and molecular features. More than 50\% of DLBCL are elderly patients older than 60 years at diagnosis [1] and have advanced stage disease, intermediate- to high-risk International Prognostic Index (IPI) and adverse clinical outcome [2-4]. The progression-free survival (PFS) and overall survival (OS) rates were only $54 \%$ and $58 \%$ at
\end{abstract}


5 years upon treatment with R-CHOP (rituximab, cyclophosphamide, doxorubicin, vincristine, and prednisone) [2], remarkably inferior to those of DLBCL patients younger than 60 years [5]. Moreover, most of the elderly patients are ineligible for intensive chemotherapy and/or hematopoietic stem cell transplantation, making effective and safe treatment as an unmet need in this subset of DLBCL.

In addition to clinical parameters, several biological features have been revealed as important prognostic indicators in DLBCL. Based on cell of origin, DLBCL can be classified as germinal center B-cell like (GCB), activated $B$-cell like $(A B C)$, and unclassified, the latter two referred as non-GCB phenotype $[6,7]$. Patients with non-GCB have a worse prognosis than those with GCB phenotype [6]. Double-expressor lymphoma (DEL) is defined as coexpression of BCL2 (>50\%) and MYC (>40\%) proteins by immunohistochemistry without underlying rearrangements [1]. DEL patients respond poorly to standard $\mathrm{R}$-CHOP or intensive chemotherapy followed by stem cell transplantation [8]. As for molecular alterations, epigenetic gene mutations are frequently observed in DLBCL, mainly including histone methyltransferases $K M T 2 C$, $K M T 2 D$, and $E Z H 2$, histone acetyltransferases CREBBP, EP300, and IRF4, chromatin remodeler HIST1H1E and ARID1A, and DNA methylation gene TET2 [9]. GCB DLBCL with CREBBP, EP300, and KMT2D mutations tends to have inferior prognosis [10]. In a SAKK 38/07 prospective cohort, $C R E B B P$ mutation is an independent prognostic factor in DLBCL [11]. Elderly DLBCL patients are generally more frequently categorized into those high-risk groups [12, 13], providing clues for searching potential targeted therapeutic strategies.

Novel agents in combination with R-CHOP have been shown to improve clinical outcome of DLBCL. Ibrutinib plus R-CHOP prolongs PFS and OS in patients younger than 60 years [14]. Lenalidomide plus R-CHOP is effective in elderly patients with acceptable tolerability and mitigates the negative impact of non-GCB phenotype on patient prognosis [15]. Histone deacetylase inhibitors (HDACIs) are potent anti-lymphoma agents and synergistic activity between HDACIs and rituximab was observed [16]. Moreover, HDACIs sensitize B-lymphoma cells to chemotherapeutic agents [17] and clinical efficacy of HDACIs has also been investigated in patients with newly diagnosed DLBCL in combination with R-CHOP $[18,19]$.

Tucidinostat (formerly known as chidamide) is an oral benzamide class of HDACI that selectively inhibits Class I HDAC1, HDAC2, HDAC3, and Class IIb HDAC10, and has been applied to treat relapsed or refractory peripheral T-cell lymphoma as mono- or combinational therapy [20]. In the present study, a phase II study of chidamide plus $\mathrm{R}-\mathrm{CHOP}$ (CR-CHOP) was conducted to evaluate the efficacy and safety in elderly patients with newly diagnosed DLBCL. Meanwhile, we performed whole genome sequencing (WGS) and targeted sequencing in patients with available tumor samples to explore relevance of molecular biomarkers in this prospective cohort.

\section{Results}

\section{Patient characteristics}

A total of 49 patients were included in the study between May 10, 2016 and May 2, 2018. Baseline characteristics are summarized in Table 1. Median age was 67 years (range 61-75), and 29 patients (59\%) were male. Forty patients (82\%) presented advanced Ann Arbor stage, and 41 patients $(84 \%)$ showed elevated serum lactate dehydrogenase (LDH) level. Twenty-nine patients (59\%) had

Table 1 Baseline clinical and pathological characteristics

\begin{tabular}{|c|c|}
\hline Characteristics & $\begin{array}{l}\text { Enrolled patients } \\
(n=49)\end{array}$ \\
\hline \multicolumn{2}{|l|}{ Age (years) } \\
\hline Median & $67(61-75)$ \\
\hline \multicolumn{2}{|l|}{ Gender } \\
\hline Male & $29(59 \%)$ \\
\hline Female & $20(41 \%)$ \\
\hline \multicolumn{2}{|l|}{ ECOG } \\
\hline $0-1$ & $38(78 \%)$ \\
\hline 2 & $11(22 \%)$ \\
\hline \multicolumn{2}{|l|}{ Ann Arbor stage } \\
\hline$\|$ & $9(18 \%)$ \\
\hline III & $15(31 \%)$ \\
\hline IV & $25(51 \%)$ \\
\hline \multicolumn{2}{|l|}{$L D H$} \\
\hline Normal & $8(16 \%)$ \\
\hline Elevated & $41(84 \%)$ \\
\hline \multicolumn{2}{|l|}{ Extranodal sites } \\
\hline $0-1$ & $20(41 \%)$ \\
\hline$\geq 2$ & $29(59 \%)$ \\
\hline \multicolumn{2}{|l|}{$|P|$} \\
\hline 2 & $7(14 \%)$ \\
\hline 3 & $17(35 \%)$ \\
\hline 4 & $20(41 \%)$ \\
\hline 5 & $5(10 \%)$ \\
\hline \multicolumn{2}{|l|}{ Cell of origin } \\
\hline GCB & $14(29 \%)$ \\
\hline non-GCB & $35(71 \%)$ \\
\hline \multicolumn{2}{|c|}{ Double expressor lymphoma } \\
\hline Yes & $12(25 \%)$ \\
\hline No & $37(75 \%)$ \\
\hline
\end{tabular}

ECOG Eastern Cooperative Oncology Group, LDH lactate dehydrogenase, IPI International Prognostic Index 
multiple extranodal involvement, mainly as bone (35\%), gastrointestinal (24\%), and bone marrow (18\%). Fortytwo patients $(86 \%)$ were of intermediate-high or high-risk of IPI at diagnosis. Twelve patients had BCL2/MYC double expression with exclusion of MYC and BCL2/BCL6 translocation.

\section{Dose intensity}

As shown in Fig. 1, 43 patients (88\%) completed all six cycles of CR-CHOP. Two patients did not continue after the first three cycles because of stable disease. One patient withdrew consent after four cycles, and another three patients discontinued after five cycles: one withdrew consent, and two stopped due to adverse events (AEs), all of whom were in complete remission.

Of the 294 planned treatment cycles, 283 (96\%) were given. The full dose was given in 266 (90\%) of the 294 planned cycles. Tucidinostat postpones were applied in 8 cycles (3\%), due to grade 4 neutropenia on day 11 . Cyclophosphamide and doxorubicin dose reductions were applied in 15 cycles (5\%), because of treatment delay due to grade 3 infections and grade 3-4 neutropenia. When analyzed by number of patients rather than number of cycles, four and eight patients had postpones of tucidinostat, and reduction of cyclophosphamide and doxorubicin, respectively.

\section{Response and survival}

After completion of stage 1, 20 (87\%, 95\% CI 72-100) of 23 patients achieved complete response, justifying the initiation of stage 2 of Simon's design. After completion of stage 2, 42 patients (86\%, 95\% CI 76-96) achieved complete response, and 4 patients $(8 \%)$ achieved partial response. The four patients with partial response received additional involved-field radiotherapy to sites of residual uptake at final positron emission tomographycomputed tomography (PET-CT), and one patient eventually achieved complete response. All three patients who did not respond after three cycles of treatment (including one patient with double hit lymphoma and one patient with triple-hit lymphoma) were salvaged with secondline chemotherapy and died from disease progression.

With a median follow-up of 30 months (range 6-42), the 2-year PFS and OS rates were $68 \%$ (95\% CI 52-79) and $83 \%$ (95\% CI 68-91), respectively (Fig. 2a). The 2-year PFS was 73\% (95\% CI 48-87) for patients with intermediate-risk (IPI 2-3) and 63\% (95\% CI 40-79) for those with high-risk (IPI 4-5) (HR 0.568, 95\% CI $0.213-$ $1.514 ; P=0.265$; Fig. 2b). The 2 -year OS was $85 \%$ (95\% CI 61-95) for patients with intermediate-risk and $80 \%(95 \%$ CI 58-91) for those with high-risk (HR 0.658, 95\% CI $0.191-2.274 ; P=0.513$; Fig. $2 b$ ).

\section{Toxicity}

Hematological and non-hematological AEs are summarized in Table 2. For hematological toxicities, grade 3 and 4 neutropenia were present in $31 \%$ and $53 \%$ of patients, respectively. Neutropenia was of short duration, with a median of 5 days (range, 3-7). Febrile neutropenia was reported in 15 cycles and was of grade 3 in maximum. Grade 3 and 4 thrombocytopenia were reported in $8 \%$ and $2 \%$ of patients, respectively, without bleeding complications. Grade 3 anemia was observed in 18\% of patients. For non-hematological toxicities, grade 3 alanine aminotransferase (ALT) or aspartate aminotransferase (AST) elevation was observed in $8 \%$ of patients. No grade 4 nonhematologic toxicities were reported. Epstein-Barr virus DNA (EBV-DNA) was monitored routinely throughout the trial, and no positive event was reported.

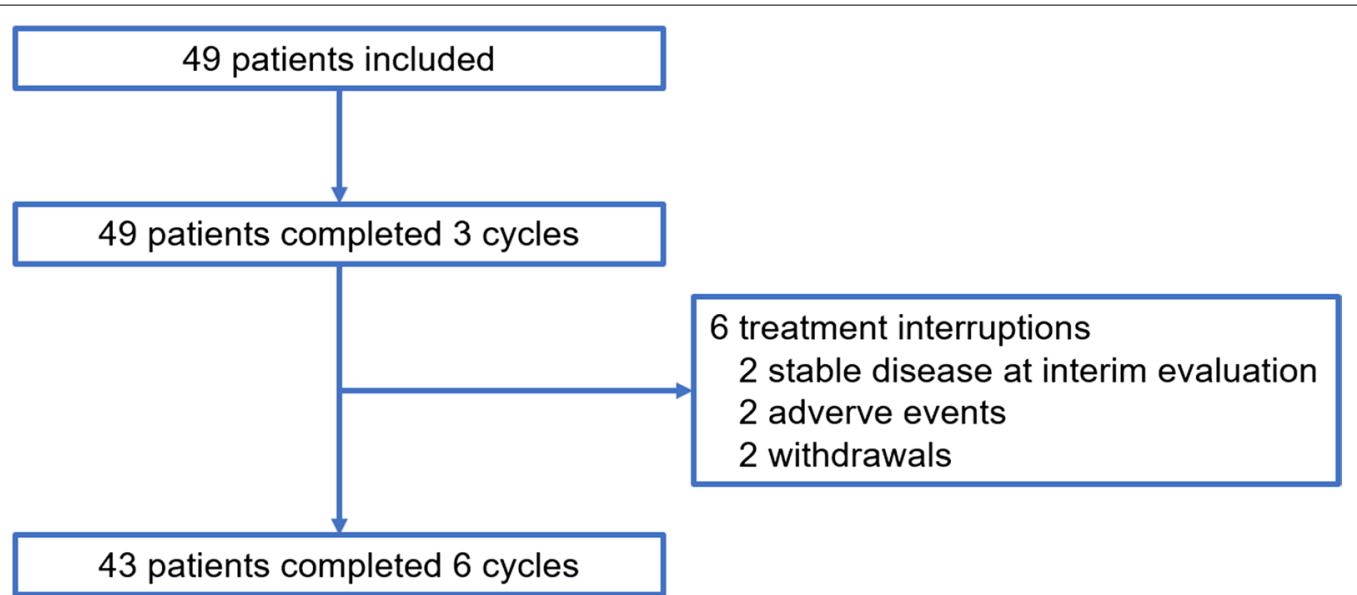

Fig. 1 CONSORT diagram of the study 


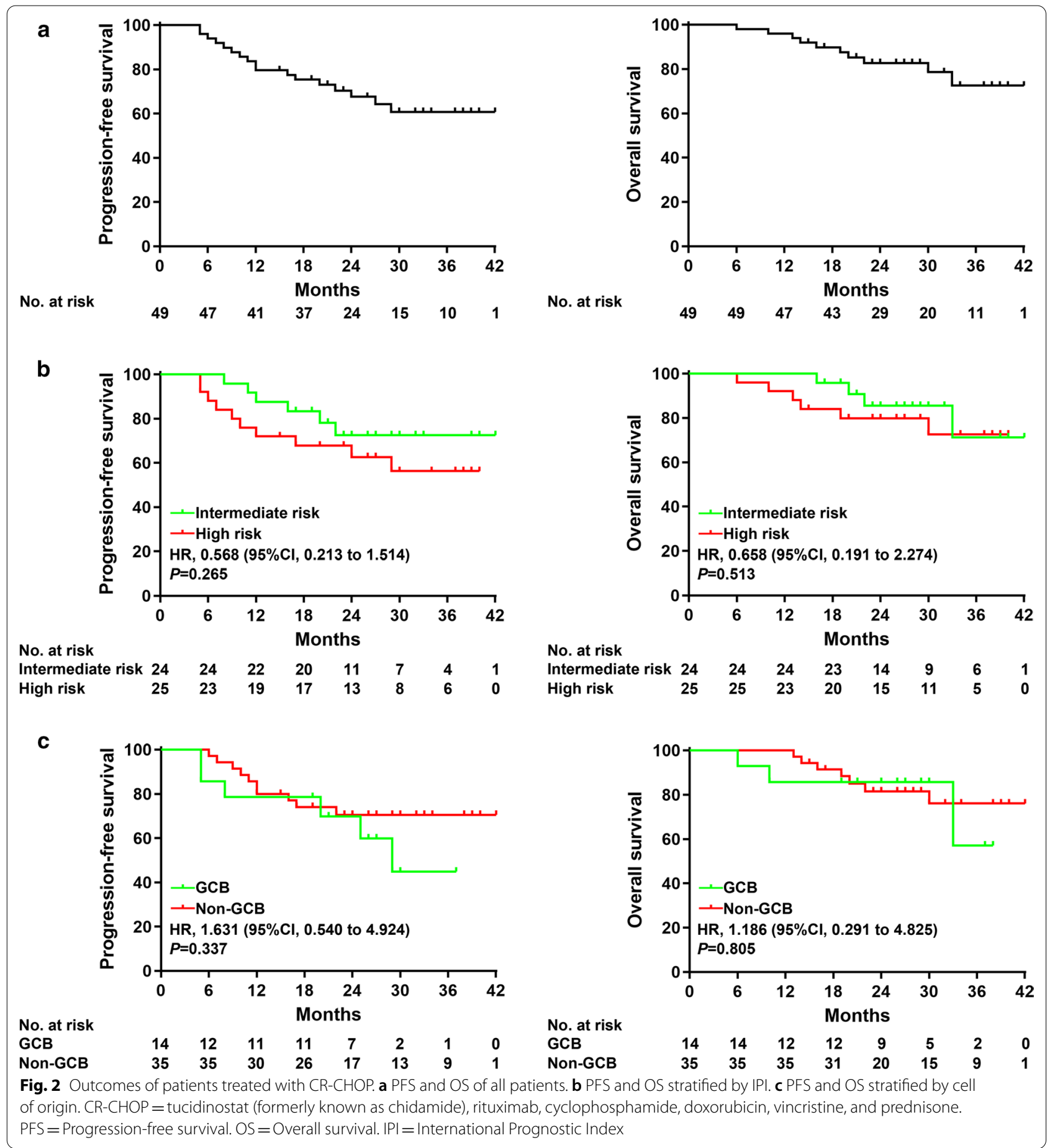

No death occurred during the study as a result of toxicity related to treatment. Overall, nine patients died: eight for lymphoma progression, and one for severe pulmonary infection and heart failure in complete remission.
Impact of phenotype on response to $\mathrm{CR}-\mathrm{CHOP}$

Twelve (86\% [95\% CI 65-100]) of the 14 patients with GCB achieved complete response, as did 30 (86\% [95\% CI 74-98]) of the 35 patients with non-GCB phenotype. The 2-year PFS was $70 \%$ (95\% CI 38-88) in the 
Table 2 Hematological and non-hematological adverse events

\begin{tabular}{|c|c|c|c|c|}
\hline Adverse events & Grade 1 & Grade 2 & Grade 3 & Grade 4 \\
\hline \multicolumn{5}{|c|}{$\begin{array}{l}\text { Number of cycles in which hematological adverse events were reported } \\
(n=283)\end{array}$} \\
\hline Neutropenia & $11(4 \%)$ & $28(10 \%)$ & $58(20 \%)$ & $113(40 \%)$ \\
\hline Thrombocytopenia & $70(25 \%)$ & $48(17 \%)$ & $17(6 \%)$ & $10(4 \%)$ \\
\hline Anemia & $85(30 \%)$ & $34(12 \%)$ & $11(4 \%)$ & 0 \\
\hline Febrile neutropenia & 0 & 0 & $15(5 \%)$ & 0 \\
\hline \multicolumn{5}{|c|}{ Number of patients with hematological adverse events $(n=49)$} \\
\hline Neutropenia & $2(4 \%)$ & $3(6 \%)$ & $15(31 \%)$ & $26(53 \%)$ \\
\hline Thrombocytopenia & $12(24 \%)$ & $10(20 \%)$ & $4(8 \%)$ & $1(2 \%)$ \\
\hline Anemia & $17(35 \%)$ & $12(24 \%)$ & $9(18 \%)$ & 0 \\
\hline Febrile neutropenia & 0 & 0 & $6(12 \%)$ & 0 \\
\hline \multicolumn{5}{|c|}{ Number of patients with non-hematological adverse events $(n=49)$} \\
\hline Liver function abnormalities & $5(10 \%)$ & $1(2 \%)$ & $4(8 \%)$ & 0 \\
\hline Infection & $2(4 \%)$ & $6(12 \%)$ & $8(16 \%)$ & 0 \\
\hline Fatigue & $2(4 \%)$ & $3(6 \%)$ & 0 & 0 \\
\hline Vomiting & $3(6 \%)$ & $1(2 \%)$ & 0 & 0 \\
\hline Diarrhea & $2(4 \%)$ & $1(2 \%)$ & 0 & 0 \\
\hline Hypoalbuminemia & $5(10 \%)$ & 0 & 0 & 0 \\
\hline Hypokalemia & $4(8 \%)$ & 0 & 0 & 0 \\
\hline Heart failure & 0 & $1(2 \%)$ & 0 & 0 \\
\hline Atrial fibrillation & 0 & $1(2 \%)$ & 0 & 0 \\
\hline Neurological & $2(4 \%)$ & 0 & 0 & 0 \\
\hline
\end{tabular}

GCB group and $71 \%$ (95\% CI 52-83) in the non-GCB group (HR 1.631, 95\% CI (0.540-4.924); $P=0.337$; Fig. 2c). The 2-year OS was $86 \%$ (95\% CI 54-96) in the GCB group and $81 \%$ (95\% CI 63-91) in the non-GCB group (HR 1.186, 95\% CI (0.291-4.825); $P=0.805$; Fig. 2c). Outcomes for non-GCB patients tended to be improved upon CR-CHOP, as compared to historical control (2-year PFS and OS of 60\% [95\% CI 49-68] and $72 \%$ [95\% CI 62-80], respectively).

All twelve patients (100\%) with DEL phenotype achieved complete response, as did 30 (86\% [95\% CI 74-98]) of the 35 patients with non-DEL phenotype. The 2-year PFS and OS rates of patients with DEL phenotype were $83 \%$ (95\% CI 48-96) and 92\% (95\% CI 54-79), which were comparable to those of nonDEL phenotype (PFS: HR 0.394, 95\% CI 0.125-1.240, $P=0.203$; OS: HR 0.307 , 95\% CI $0.069-1.362, P=0.232$; Fig. 3a). Instead, in historical control (NCT01852435) upon R-CHOP treatment, the 2-year PFS and OS rates of patients with DEL phenotype were 46\% (95\% CI 31-61) and 63\% [95\% CI 46-76], which were significantly lower than those of non-DEL phenotype (PFS: HR 2.041, 95\% CI 1.111-3.750, $P=0.010$; OS: HR 2.249, 95\% CI 1.166-4.338, $P=0.008$; Fig. 3b).
Impact of molecular alterations on response to $\mathrm{CR}-\mathrm{CHOP}$ As shown in Fig. 4a, epigenetic gene mutations were identified in 21 of $36(58 \%)$ patients, including genes related to histone methylation (KMT2D [5/36, 14\%], $K M T 2 C$ [2/36, 6\%], and $E Z H 2$ [5/36, 14\%]), histone acetylation (CREBBP [3/36, 8\%], EP300 [2/36, 6\%], and IRF4 [1/36, 3\%]), chromatin remodeler (HIST1H1E [8/36, 22\%] and ARID1A [1/36, 3\%]), and DNA methylation (TET2 [8/36, 22\%]). Overall, a total of 47 non-silent somatic mutations were identified, including 34 missense, 9 insertion or deletion, 4 nonsense, and a preference for $\mathrm{C}>\mathrm{T} / \mathrm{A}>\mathrm{G}$ alterations analogous to the somatic single nucleotide variation (SNV) spectrum in other cancers.

In terms of the influence of epigenetic gene mutations on clinical outcome, mutations in KMT2D, but not in $C R E B B P / E P 300$, were associated with inferior PFS and OS ( $P=0.043$ and $<0.001$, respectively, Fig. $4 \mathrm{~b})$. The 2-year PFS and OS rates were $40 \%$ (95\% CI 5-75) and $40 \%$ (95\% CI 5-75) in patients with KMT2D mutation, significantly shorter than those without mutation (68\% [95\% CI 47-82] and 89\% [95\% CI 70-96]). Although mutations in CREBBP/EP300 were associated with inferior PFS and OS in patients of historical control (mutation rate 15\%, PFS: HR 3.292, 95\% CI 1.1409.502, $P=0.028$; OS: HR 4.628, 95\% CI 1.379-15.530, $P=0.013)$, there was no significant difference of PFS and OS between patients with or without $C R E B B P / E P 300$ mutation upon treatment with CR-CHOP $(P=0.426$ and 0.971, respectively; Fig. 4b). The 2-year PFS and OS rates were $80 \%$ (95\% CI 20-97) and 80\% (95\% CI 20-97) in patients with CREBBP/EP300 mutation, 61\% (95\% CI $40-87)$ and $83 \%$ (95\% CI 63-92) in patients without $C R E B B P / E P 300$ mutation, respectively.

\section{Discussion}

DLBCL in the elderly is associated with adverse clinical outcome and limited treatment options. In this phase II study, we showed that CR-CHOP was effective in firstline therapy of elderly patients with newly diagnosed DLBCL. After six cycles of CR-CHOP, the complete response rate was higher than those of previous reports in Western countries (71-76\%) [2, 3] and in China (72\%) [13], all of which contained low-risk IPI patients. In a recent phase I study of valproate plus $\mathrm{R}-\mathrm{CHOP}$ in newly diagnosed DLBCL (26\% of the patients had intermediatehigh or high-risk IPI), improvement of clinical outcome has been achieved, with PFS of $84.7 \%$ and OS of $96.8 \%$ at 2 years [18]. The 2-year survival time was also encouraging with our study on CR-CHOP, considering that more than $80 \%$ of patients had intermediate-high or high-risk IPI. Of note, CR-CHOP was equally effective in highrisk IPI patients as intermediate-high IPI patients, and 


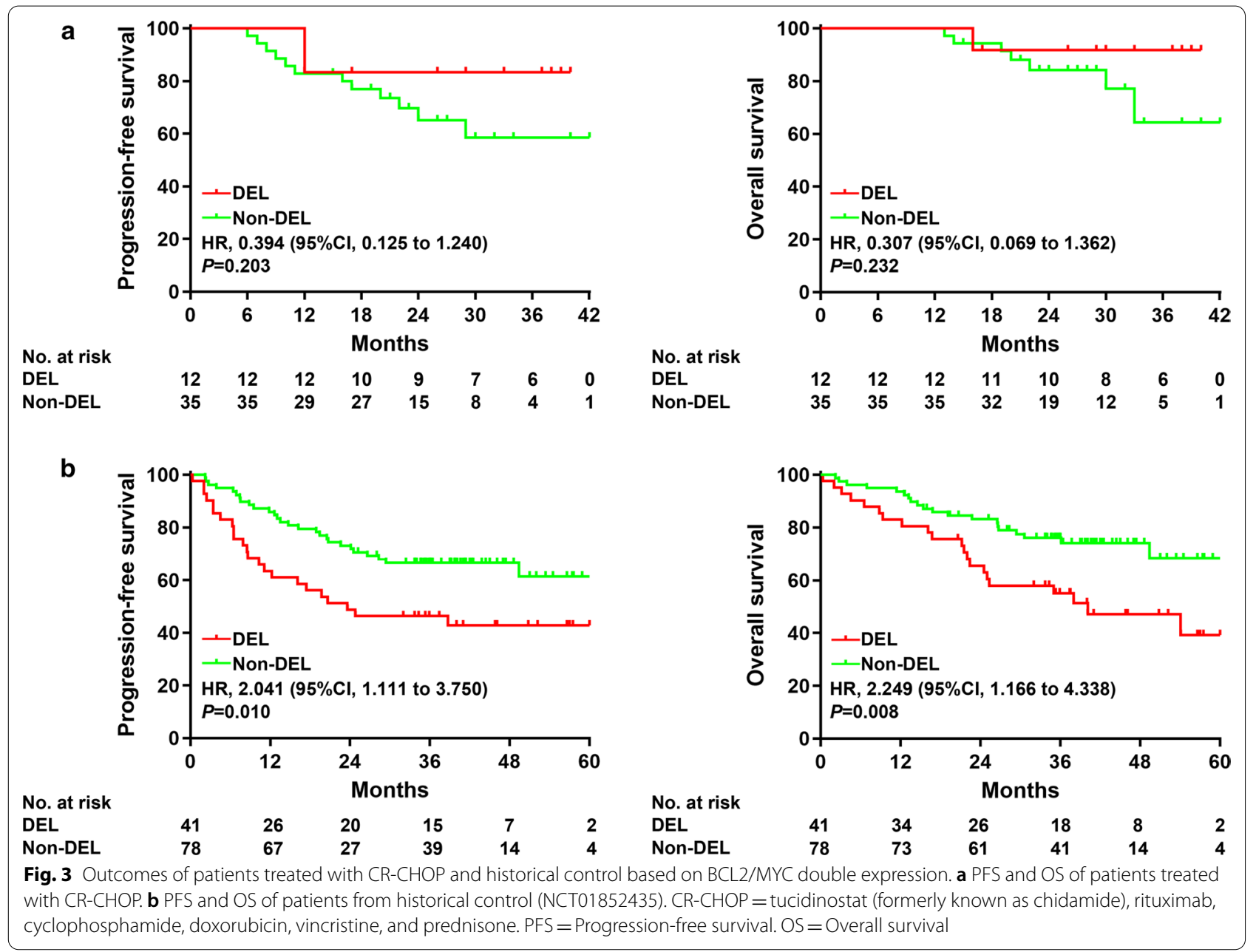

compared favorably to standard $\mathrm{R}-\mathrm{CHOP}$ reported by LNH985 (2-year PFS and OS as approximately 50\% and $60 \%$ ) [2] and by our previous study (2-year PFS and OS as $41 \%$ and $63 \%$, respectively) [13].

Another encouraging finding of our study was the clinical efficacy of CR-CHOP in the DEL phenotype of DLBCL. Though in historical control (NCT01852435), the outcome of DEL patients was significantly inferior to that of non-DEL patients upon R-CHOP treatment, no apparent difference was observed in patients treated with CR-CHOP. Improved outcomes were also reported for DEL in a phase I/II trial of vorinostat plus R-CHOP (SWOG S0806), as compared to those of R-CHOP (SWOG S0433) (2-year PFS $73 \%$ vs. 58\%, 2-year OS $91 \%$ vs. $75 \%$ ) [19]. These clinical findings are supported by experimental data showing that MYC acts as a biomarker of the anti-cancer action of HDACI romidepsin and entinostat in DLBCL $[21,22]$. In other hematological malignancies, tucidinostat inhibits MYC and BCL2 in acute myeloid leukemia [23, 24], and tucidinostat also downregulates BCL2 in combination with doxorubicin in peripheral T-cell lymphoma [25]. Together, tucidinostat may improve the outcome of DLBCL patients through targeting MYC and BCL2. To further confirm the role of tucidinostat plus R-CHOP in DEL phenotype of DLBCL patients, a phase III, randomized, double-blind, placebocontrolled, multicenter study is currently ongoing in China (NCT04231448).

Histone modifying genes are critically involved in tumor progression of DLBCL [26]. Inactivation of $C R E B B P$ and EP300 abrogates germinal center $\mathrm{B}$ cell formation and contributes to lymphomagenesis, as revealed by conditional germinal center-directed deletion mouse models targeting Crebbp or Ep300 [27]. Clinically, CREBBP mutation is an independent prognostic factor in DLBCL in a prospective SAKK 38/07 trial [11]. Disruption of $K M T 2 D$ also perturbs germinal center B cell development $[28,29]$ and $K M T 2 D$ mutations are the most frequent relapse-specific events in DLBCL [30]. In consistence with experimental data 


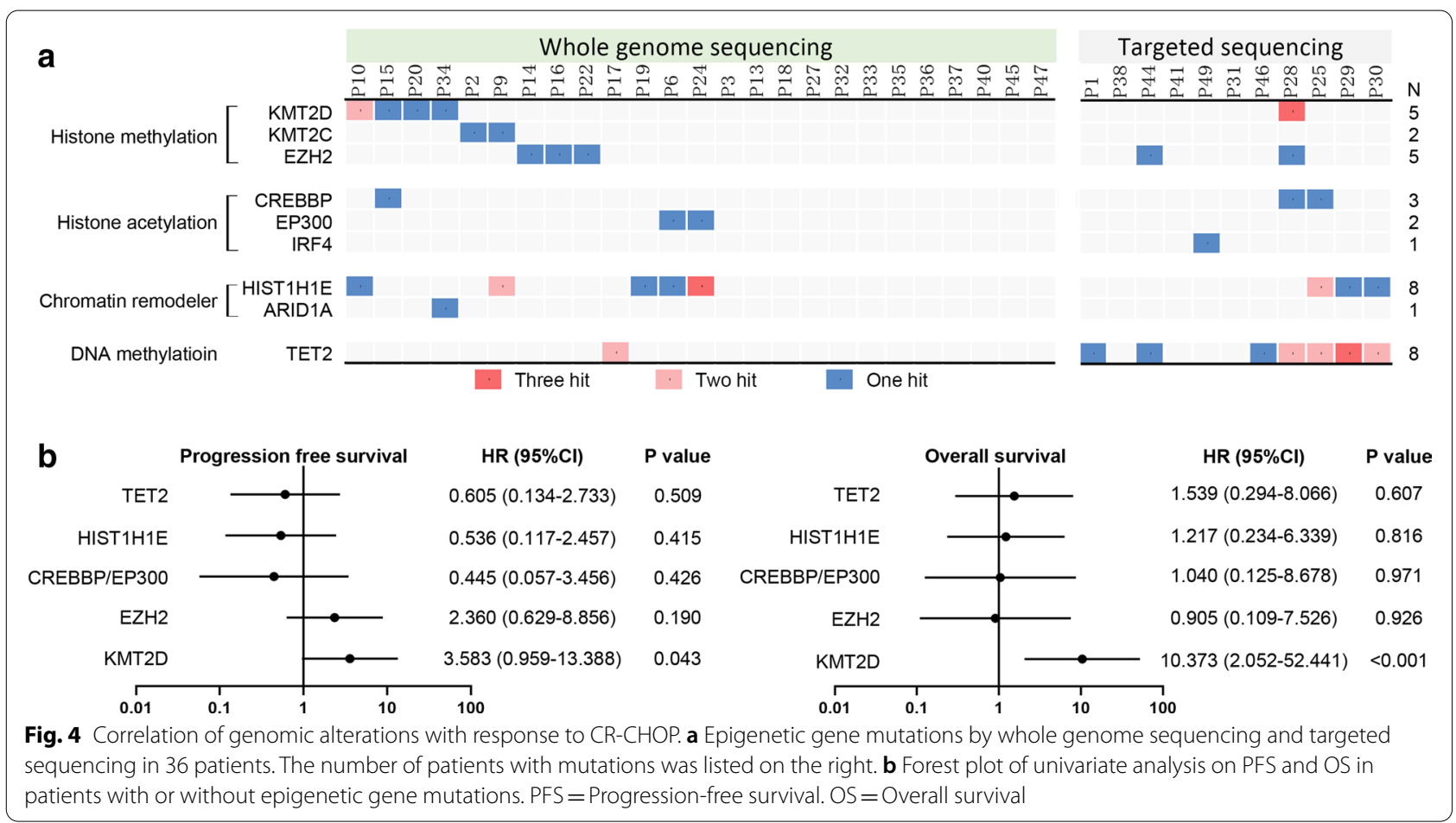

showing that HDACIs can rescue deficits in histone acetylation induced by $C R E B B P / E P 300$ mutations in B-cell lymphoma [26], our results provided clinical evidence that tucidinostat may mitigate the negative prognostic impact of CREBBP/EP300 mutations on DLBCL. However, mutations in KMT2D remained to be an adverse prognostic factor in DLBCL treated with CR$\mathrm{CHOP}$, which could be alternatively targeted by hypomethylating agents [31].

Safety profile is a major issue for the addition of novel agent to an existing combination, including HDACIs. Vorinostat plus R-CHOP is associated with high rates of AEs in patients with newly diagnosed advanced stage DLBCL (SWOG S0806) [19]. Also, unexpected auditory AEs occur in valproate plus R-CHOP [18]. Here, we recorded no grade 4 non-hematological AEs and no deaths due to toxicity. Grade 3-4 neutropenia was recorded in almost $20 \%-40 \%$ of cycles despite the use of granulocyte-colony stimulating factor (G-CSF), but it was of short duration and did not translate into an excess of infections. Most AEs in our trial were of mildto-moderate intensity, and similar to those recorded with standard R-CHOP in elderly patients with DLBCL. No new clinically significant toxicity was noted with the addition of tucidinostat to R-CHOP. EBV reactivation is of concern in the administration of HDACI [32] and was not observed in this study.
With regard to dose intensity and the feasibility of the CR-CHOP regimen, the analysis of drug delivery showed that patients received full dose of tucidinostat and at least $90 \%$ of the doses of R-CHOP drugs in more than $90 \%$ of cycles. This frequency is acceptable when comparing with other studies of standard R-CHOP, in which the percentage of the patients who received more than $90 \%$ of the dose intensity of the drugs varied from 64 to $90 \%$ $[14,15]$. Therefore, the addition of tucidinostat does not impair full delivery of R-CHOP, provided that G-CSF is used.

\section{Conclusion}

CR-CHOP was well tolerated and showed promising clinical activity in DLBCL. Our encouraging data warrant validation in a future phase III randomized trial to elucidate the relevance of DEL phenotype and molecular biomarkers on response to $\mathrm{R}-\mathrm{CHOP}$ in combination with tucidinostat in DLBCL.

\section{Methods}

\section{Eligibility criteria}

Patients were eligible if they were 61-75 years; had newly diagnosed, histologically confirmed CD20-positive DLBCL, an Eastern Cooperative Oncology Group (ECOG) performance status of $0-2$, IPI risk of intermediate or high (IPI $\geq 2$ ), and a life expectancy of more 
than 6 months. Patients were excluded if they had previous chemotherapy or stem cell transplantation; history of malignancy (other than skin cancers or carcinomas in situ of the cervix); uncontrollable cardio-cerebral vascular, coagulation, autoimmune, infectious disease; primary central nervous system (CNS) lymphoma; left ventricular ejection fraction $\leq 50 \%[4,33]$. They were also eliminated from the study if, at enrollment, their neutrophil count $<1.5 \times 10^{9} / \mathrm{L}$, platelets $<75 \times 10^{9} / \mathrm{L}$, ALT or AST $>2 \times$ upper limit of normal (ULN), AKP or bilirubin $>1.5 \times$ ULN, or creatinine $>1.5 \times$ ULN (unless they were caused by the lymphoma). They were not enrolled if they were not able to comply with the protocol for mental or other unknown reasons; pregnancy or lactation; or positive for hepatitis B virus (HBV-DNA) and human immunodeficiency virus.

Pathological diagnosis was performed according to the 2016 World Health Organization classification [1]. Cell of origin profile was determined by Hans algorithm, with $30 \%$ cutoff value of CD10, BCL6, and MUM-1 [6]. As for DEL phenotype, cutoff value of BCL2 and MYC was 50\% and $40 \%$, respectively [1]. Fluorescence in situ hybridization of BCL2, BCL6, and MYC rearrangements was performed for each patient.

\section{Study design and procedures}

This was an investigator-initiated, open-label, single-arm, phase II study. The dose and administration schedule of CR-CHOP were as follows: rituximab $375 \mathrm{mg} / \mathrm{m}^{2}$ given intravenously on day 0 , cyclophosphamide $750 \mathrm{mg} / \mathrm{m}^{2}$, doxorubicin $50 \mathrm{mg} / \mathrm{m}^{2}$, and vincristine $1.4 \mathrm{mg} / \mathrm{m}^{2}$ (maximum $2.0 \mathrm{mg}$ ) intravenously on day 1 , prednisone $60 \mathrm{mg} /$ $\mathrm{m}^{2}$ (maximum $100 \mathrm{mg}$ ) orally on days $1-5$, and tucidinostat $20 \mathrm{mg}$ orally on days $1,4,8$, and 11 , according to the maximum tolerated dose in combination with $\mathrm{CHOP}$ and etoposide in peripheral T-cell lymphoma (ClinicalTrials.gov Identifier: NCT02987244) [34]. The regimen was repeated every 21 days with a total of six cycles.

Tucidinostat should be postponed on the occurrence of grade $\geq 3$ hematological or non-hematological toxicities. There was no plan for dose reduction of tucidinostat. The administration was resumed when the AEs were to grade 1 or pre-treatment levels. G-CSF prophylaxis (recombinant human pegylated G-CSF of $100 \mathrm{ug} / \mathrm{kg}$ ) was given from the second cycle of chemotherapy if grade $\geq 3$ neutropenia was present in the first cycle. Lamivudine was administered in occult carriers of HBV to prevent HBV reactivation. Prophylaxis for CNS relapse was given to patients with involvement of bone marrow, nasal or paranasal sinuses, orbit, breast, or testis. Tumor lysis prophylaxis and radiation therapy were performed for patients with bulky disease, or with residual disease at the end of treatment, at the discretion of physicians.
Baseline evaluations were performed within 28 days prior to therapy, including physical examination, complete blood cell count, serum biochemistry with LDH, coagulation function, HBV markers and DNA, EBVDNA, electrocardiogram, echocardiography, bone marrow aspiration and trephine biopsy, and PET-CT. The patients were staged according to Ann Arbor staging system, and IPI [35] was calculated. Treatment response was assessed according to the standardized response criteria established by Cheson et al. [36]. Interim efficacy was evaluated by PET-CT after three cycles. Patients who had achieved a complete or partial response received another three cycles. Patients who did not achieve a complete or partial response stopped receiving CR-CHOP at this point. Final evaluation was performed by PET-CT one month after the end of the last cycle of treatment. CT of the neck, thorax, abdomen, and pelvis was repeated every 3 months thereafter to monitor disease progression until 1 year, then every 6 months until 2 years, and every year thereafter.

\section{Study end points and assessments}

The primary endpoint was complete response rate assessed by PET-CT. Secondary endpoints were PFS, OS, overall response rate, and AEs. PFS was measured from diagnosis to date of progression, relapse, or death from any cause. Patients with a partial response who were given an additional treatment (i.e., radiation therapy) without apparent disease progression were not considered as an event for PFS analysis. OS was measured from diagnosis to death of any cause or date of last followup. AEs were categorized and graded according to the National Cancer Institute Common Terminology Criteria for Adverse Events (CTCAE, version 4.0).

\section{Historical control cohort}

Elderly patients from 20 centers of the Multicenter Hematology/Oncology Programs Evaluation System (M-HOPES) in China with newly diagnosed DLBCL, treated with regular R-CHOP50 (doxorubicin $50 \mathrm{mg} /$ $\mathrm{m}^{2}$ ) or regular R-CEOP70 (rituximab, cyclophosphamide, epirubicin $70 \mathrm{mg} / \mathrm{m}^{2}$, vincristine, and prednisone) between May 15, 2013 and March 16, 2016 in our previous multicenter, phase III, randomized, controlled trial (NCT01852435) [13], and who met the same inclusion/ exclusion criteria as those treated with CR-CHOP were referred as the historical control cohort and analyzed for outcome on the basis of DLBCL subtypes.

\section{DNA sequencing}

WGS was performed on frozen tumor samples of 25 patients with genomic DNA extracted using Wiz$\operatorname{ard}^{\circledR}$ Genomic DNA Purification Kit (Promega, 
Wisconsin-Madison, USA). The depth of samples measured with WGS was $50-200 \times$, with $83-99 \%$ of the target sequence being covered sufficiently deep for variant calling ( $\geq 10 \times$ coverage). A total of 9 genes reported to be hypermutation $(>5 \%)$ or related to lymphoma pathogenesis in DLBCL were selected. SNVs and indels were called by Genome Analysis Toolkit (GATK, v3.7.0) Haplotype Caller and GATK Unified Genotyper, and mapped to the genome location using the UCSC Genome Browser (https://genome.ucsc.edu/). The reference genome was the Refseq database (Human Reference Genome version hg19). All the somatic functional mutations, including non-synonymous SNVs, frameshift or in-frame indels, stopgain or stoploss, were obtained. Visual inspection was used to exclude potential false positive results. Homemade pipeline was used to filter SNVs and indels detected by the above software, excluding: (1) mutations reported with low confidence; (2) germline mutations detected from control samples; (3) population-related variants reported in 1000 Genomes (dbSNP 138) as common SNPs and not included in COSMIC (the Catalogue of Somatic Mutations in Cancer) version v77.

Targeted sequencing was performed on formalin-fixed paraffin-embedded tumor samples of 11 patients with genomic DNA extracted using GeneRead DNA formalin-fixed paraffin-embedded Tissue Kit (Qiagen, Hilden, Germany). The depth of samples measured with targeted sequencing was $1000-2000 \times$, with $85-98 \%$ of the target sequence being covered sufficiently deep for variant calling $(\geq 10 \times$ coverage).

\section{Statistical analysis}

This phase II study was designed according to Simon's two-stage minimax design [37]. Our objective was to show a $15 \%$ improvement in frequency of complete response with the new regimen relative to an expected frequency of $70 \%$ with R-CHOP alone [38], with an $\alpha$ of $0.05,80 \%$ power. A total of 49 patients were required to obtain the hypothesis, 23 of whom were to be enrolled during stage 1 and 26 during stage 2 . The study would be stopped early if fewer than $16(70 \%)$ of the patients achieved complete response in stage 1. Similarly, if 39 $(80 \%)$ or fewer patients achieved complete response by trial completion, the hypothesis would also be rejected.

Efficacy and safety analyses were by intention to treat. Statistical analyses were performed by Statistical Package for the Social Science (SPSS) 23.0 software (SPSS Inc., Chicago, IL, USA). Survival estimates were calculated by Kaplan-Meier method, and survival curves were compared by log-rank test. A two-sided P value of $<0.05$ was considered statistically significant.

\section{Abbreviations}

DLBCL: Diffuse large B-cell lymphoma; IPI: International Prognostic Index; PFS: Progression-free survival; OS: Overall survival; R-CHOP: Rituximab, cyclophosphamide, doxorubicin, vincristine, and prednisone; GCB: Germinal center B-cell; ABC: Activated B-cell; DEL: Double-expressor lymphoma; HDACl: Histone deacetylase inhibitor; CR-CHOP: Tucidinostat (formerly known as Chidamide), rituximab, cyclophosphamide, doxorubicin, vincristine, and prednisone; WGS: Whole genome sequencing; LDH: Lactate dehydrogenase; AE: Adverse event; PET-CT: Positron emission tomography-computed tomography; ALT: Alanine aminotransferase; AST: Aspartate aminotransferase; EBV-DNA: EpsteinBarr virus DNA; SNV: Single nucleotide variation; G-CSF: Granulocyte-colony stimulating factor; ECOG: Eastern Cooperative Oncology Group; CNS: Central nervous system; ULN: Upper limit of normal; HBV-DNA: Hepatitis B virus DNA; CTCAE: Common Terminology Criteria for Adverse Events.

\section{Acknowledgements}

We appreciate the effort the physicians for enrolling patients and thank all the patients involved for allowing us to analyze their clinical data.

\section{Authors' contributions}

L.W., P.P.X, W.-L.Z. conceived and designed the study. M.-C.Z., L.W., S.C., Y.H., C.-F.W., X.-F.J., Q.S., P.-P.X., and W.-L.Z. provided executive support, active data surveillance, and performed patient selection process. M.-C.Z., Y.F., L.W., S.C., D.F., Y.Z, and P.-P.X. collected data and provided data management. M.-C.Z., D.F. P.-P.X., and W.-L.Z. performed data analysis. M.-C.Z., P.P.X., and W.-L.Z. interpreted the results and wrote the manuscript. All authors reviewed the manuscript critically and approved the content. All authors read and approved the final manuscript.

\section{Funding}

This study was supported, in part, by research funding from the National Natural Science Foundation of China (81520108003, 81830007, 81670176, and 82070204), Chang Jiang Scholars Program, Shanghai Municipal Education Commission Gaofeng Clinical Medicine Grant Support (20152206 and 20152208), Clinical Research Plan of Shanghai hospital development center (SHDC, 16CR2017A), Multicenter Clinical Research Project by Shanghai Jiao Tong University School of Medicine (DLY201601), Collaborative Innovation Center of Systems Biomedicine, and the Samuel Waxman Cancer Research Foundation. The funding source had no involvement in the study, manuscript preparation, or decision to submit the study for publication. The corresponding author had full access to all the data in the study and had final responsibility for the decision to submit for publication.

\section{Availability of data and materials}

The datasets used and/or analyzed during the current study are available in Mendeley Data through the https://doi.org/10.17632/nmxgxsvbk5.1 and National Omics Data Encyclopedia (NODE, https://www.biosino.org/node/) under Accession Number OEP001040.

\section{Ethics approval and consent to participate}

The final protocol, amendments, informed consent, and any other appropriate documents were reviewed and approved by the ethics committee and institutional review board of Shanghai Ruijin Hospital (Reference Number 2016-24). Trial registration: ClinicalTrial.gov, NCT02753647. Registered on April 28, 2016, https://clinicaltrials.gov/ct2/show/NCT02753647?term=NCT02 $753647 \&$ draw $=2 \&$ rank $=1$. The study was conducted in accordance with the principles of the Declaration of Helsinki. Written informed consent was obtained from all patients prior to enrollment.

\section{Consent for publication}

Not applicable.

\section{Competing interests}

All authors declare that they have no competing interests.

\footnotetext{
Author details

1 Shanghai Institute of Hematology, State Key Laboratory of Medical Genomics, National Research Center for Translational Medicine At Shanghai, Ruijin Hospital Affiliated To Shanghai Jiao Tong University School of Medicine, Shanghai, China. ${ }^{2}$ Pôle de Recherches Sino-Français en Science du Vivant Et Génomique, Laboratory of Molecular Pathology, Shanghai, China.
} 
${ }^{3}$ Department of Pathology, Ruijin Hospital Affiliated To Shanghai Jiao Tong University School of Medicine, Shanghai, China. ${ }^{4}$ Department of Nuclear Medicine, Ruijin Hospital Affiliated To Shanghai Jiao Tong University School of Medicine, Shanghai, China. ${ }^{5}$ Department of Radiology, Ruijin Hospital Affiliated To Shanghai Jiao Tong University School of Medicine, Shanghai, China.

Received: 31 March 2020 Accepted: 13 October 2020

Published online: 23 October 2020

\section{References}

1. Swerdlow SH, Campo E, Pileri SA, Harris NL, Stein H, Siebert R, Advani R, Ghielmini M, Salles GA, Zelenetz AD, et al. The 2016 revision of the World Health Organization classification of lymphoid neoplasms. Blood. 2016;127(20):2375-90.

2. Feugier $P$, Van Hoof A, Sebban C, Solal-Celigny P, Bouabdallah R, Ferme C, Christian B, Lepage E, Tilly H, Morschhauser F, et al. Long-term results of the R-CHOP study in the treatment of elderly patients with diffuse large B-cell lymphoma: a study by the Groupe d'Etude des Lymphomes de I'Adulte. J Clin Oncol. 2005;23(18):4117-26.

3. Pfreundschuh M, Schubert J, Ziepert M, Schmits R, Mohren M, Lengfelder E, Reiser M, Nickenig C, Clemens M, Peter N, et al. Six versus eight cycles of bi-weekly CHOP-14 with or without rituximab in elderly patients with aggressive CD20+ B-cell lymphomas: a randomised controlled trial (RICOVER-60). Lancet Oncol. 2008;9(2):105-16.

4. Cunningham D, Hawkes EA, Jack A, Qian W, Smith P, Mouncey P, Pocock C, Ardeshna KM, Radford JA, McMillan A, et al. Rituximab plus cyclophosphamide, doxorubicin, vincristine, and prednisolone in patients with newly diagnosed diffuse large B-cell non-Hodgkin lymphoma: a phase 3 comparison of dose intensification with 14-day versus 21-day cycles. Lancet. 2013;381(9880):1817-26

5. Pfreundschuh M, Kuhnt E, Trumper L, Osterborg A, Trneny M, Shepherd L, Gill DS, Walewski J, Pettengell R, Jaeger U, et al. CHOP-like chemotherapy with or without rituximab in young patients with good-prognosis diffuse large-B-cell lymphoma: 6-year results of an open-label randomised study of the MabThera International Trial (MInT) Group. Lancet Oncol. 2011;12(11):1013-22.

6. Hans CP, Weisenburger DD, Greiner TC, Gascoyne RD, Delabie J, Ott G, Muller-Hermelink HK, Campo E, Braziel RM, Jaffe ES, et al. Confirmation of the molecular classification of diffuse large B-cell lymphoma by immunohistochemistry using a tissue microarray. Blood. 2004;103(1):275-82.

7. Alizadeh AA, Eisen MB, Davis RE, Ma C, Lossos IS, Rosenwald A, Boldrick JC, Sabet $H$, Tran T, Yu X, et al. Distinct types of diffuse large B-cell lymphoma identified by gene expression profiling. Nature. 2000;403(6769):503-11.

8. Riedell PA, Smith SM. Double hit and double expressors in lymphoma: definition and treatment. Cancer. 2018;124(24):4622-32.

9. Lunning MA, Green MR. Mutation of chromatin modifiers; an emerging hallmark of germinal center B-cell lymphomas. Blood Cancer J. 2015;5:e361.

10. Schmitz R, Wright GW, Huang DW, Johnson CA, Phelan JD, Wang $J \mathrm{Q}$, Roulland S, Kasbekar M, Young RM, Shaffer AL, et al. Genetics and pathogenesis of diffuse large B-cell lymphoma. N Engl J Med. 2018;378(15):1396-407.

11. Juskevicius D, Jucker D, Klingbiel D, Mamot C, Dirnhofer S, Tzankov A. Mutations of CREBBP and SOCS1 are independent prognostic factors in diffuse large B cell lymphoma: mutational analysis of the SAKK 38/07 prospective clinical trial cohort. J Hematol Oncol. 2017;10(1):70.

12. Rosenwald A, Wright G, Chan WC, Connors JM, Campo E, Fisher RI, Gascoyne RD, Muller-Hermelink HK, Smeland EB, Giltnane JM, et al. The use of molecular profiling to predict survival after chemotherapy for diffuse large-B-cell lymphoma. N Engl J Med. 2002;346(25):1937-47.

13. Xu PP, Fu D, Li JY, Hu JD, Wang X, Zhou JF, Yu H, Zhao X, Huang YH, Jiang L, et al. Anthracycline dose optimisation in patients with diffuse large B-cell lymphoma: a multicentre, phase 3, randomised, controlled trial. Lancet Haematol. 2019;6(6):e328-37.

14. Younes A, Sehn LH, Johnson P, Zinzani PL, Hong X, Zhu J, Patti C, Belada D, Samoilova O, Suh C et al. Randomized phase III trial of ibrutinib and rituximab plus cyclophosphamide, doxorubicin, vincristine, and prednisone in Non-Germinal Center B-cell diffuse large B-cell lymphoma. J Clin Oncol. 2019:Jco1802403.

15. Vitolo U, Chiappella A, Franceschetti S, Carella AM, Baldi I, Inghirami G, Spina M, Pavone V, Ladetto M, Liberati AM, et al. Lenalidomide plus R-CHOP21 in elderly patients with untreated diffuse large B-cell lymphoma: results of the REAL07 open-label, multicentre, phase 2 trial. Lancet Oncol. 2014;15(7):730-7.

16. Zhao WL, Wang L, Liu YH, Yan JS, Leboeuf C, Liu YY, Wu WL, Janin A, Chen Z, Chen SJ. Combined effects of histone deacetylase inhibitor and rituximab on non-Hodgkin's B-lymphoma cells apoptosis. Exp Hematol. 2007;35(12):1801-11.

17. Xue K, Gu JJ, Zhang Q, Mavis C, Hernandez-Ilizaliturri FJ, Czuczman MS, Guo Y. Vorinostat, a histone deacetylase (HDAC) inhibitor, promotes cell cycle arrest and re-sensitizes rituximab- and chemo-resistant lymphoma cells to chemotherapy agents. J Cancer Res Clin Oncol. 2016;142(2):379-87.

18. Drott $K$, Hagberg H, Papworth K, Relander T, Jerkeman M. Valproate in combination with rituximab and $\mathrm{CHOP}$ as first-line therapy in diffuse large B-cell lymphoma (VALFRID). Blood Adv. 2018;2(12):1386-92.

19. Persky DO, Li H, Rimsza LM, Barr PM, Popplewell LL, Bane CL, Von Gehr A, LeBlanc M, Fisher RI, Smith SM, et al. A phase I/II trial of vorinostat (SAHA) in combination with rituximab-CHOP in patients with newly diagnosed advanced stage diffuse large B-cell lymphoma (DLBCL): SWOG S0806. Am J Hematol. 2018;93(4):486-93.

20. Shi Y, Jia B, Xu W, Li W, Liu T, Liu P, Zhao W, Zhang H, Sun X, Yang H, et al. Chidamide in relapsed or refractory peripheral $T$ cell lymphoma: a multicenter real-world study in China. J Hematol Oncol. 2017;10(1):69.

21. Shin DY, Kim A, Kang HJ, Park S, Kim DW, Lee SS. Histone deacetylase inhibitor romidepsin induces efficient tumor cell lysis via selective down-regulation of LMP1 and c-myc expression in EBV-positive diffuse large B-cell lymphoma. Cancer Lett. 2015;364(2):89-97.

22. Frys S, Simons Z, Hu Q, Barth MJ, Gu JJ, Mavis C, Skitzki J, Song L, Czuczman MS, Hernandez-Ilizaliturri FJ. Entinostat, a novel histone deacetylase inhibitor is active in B-cell lymphoma and enhances the anti-tumour activity of rituximab and chemotherapy agents. Br J Haematol. 2015;169(4):506-19.

23. Li Y, Chen $K$, Zhou Y, Xiao Y, Deng M, Jiang Z, Ye W, Wang X, Wei X, Li $J$, et al. A new strategy to target acute myeloid leukemia stem and progenitor cells using chidamide, a histone deacetylase inhibitor. Curr Cancer Drug Targets. 2015;15(6):493-503.

24. Zhao S, Guo J, Zhao Y, Fei C, Zheng Q, Li X, Chang C. Chidamide, a novel histone deacetylase inhibitor, inhibits the viability of MDS and AML cells by suppressing JAK2/STAT3 signaling. Am J Transl Res. 2016;8(7):3169-78.

25. Zhang H, Dong L, Chen Q, Kong L, Meng B, Wang H, Fu K, Wang X, PanHammarstrom Q, Wang P, et al. Synergistic antitumor effect of histone deacetylase inhibitor and Doxorubicin in peripheral T-cell lymphoma. Leuk Res. 2017;56:29-35.

26. Jiang Y, Ortega-Molina A, Geng H, Ying HY, Hatzi K, Parsa S, McNally $D$, Wang $L$, Doane AS, Agirre $X$, et al. CREBBP inactivation promotes the development of HDAC3-dependent lymphomas. Cancer Discov. 2017;7(1):38-53.

27. Meyer SN, Scuoppo C, Vlasevska S, Bal E, Holmes AB, Holloman M, Garcia-lbanez L, Nataraj S, Duval R, Vantrimpont T, et al. Unique and shared epigenetic programs of the CREBBP and EP300 acetyltransferases in Germinal Center B cells reveal targetable dependencies in Iymphoma. Immunity. 2019;51(3):535-47.e9.

28. Zhang J, Dominguez-Sola D, Hussein S, Lee JE, Holmes AB, Bansal M, Vlasevska S, Mo T, Tang H, Basso K, et al. Disruption of KMT2D perturbs germinal center $\mathrm{B}$ cell development and promotes lymphomagenesis. Nat Med. 2015;21(10):1190-8.

29. Ortega-Molina A, Boss IW, Canela A, Pan H, Jiang Y, Zhao C, Jiang M Hu D, Agirre X, Niesvizky I, et al. The histone lysine methyltransferase KMT2D sustains a gene expression program that represses B cell lymphoma development. Nat Med. 2015;21(10):1199-208.

30. Juskevicius D, Lorber T, Gsponer J, Perrina V, Ruiz C, Stenner-Liewen F, Dirnhofer S, Tzankov A. Distinct genetic evolution patterns of relapsing diffuse large B-cell lymphoma revealed by genome-wide copy number aberration and targeted sequencing analysis. Leukemia. 2016;30(12):2385-95. 
31. Ji MM, Huang YH, Huang JY, Wang ZF, Fu D, Liu H, Liu F, Leboeuf C, Wang $L$, Ye J, et al. Histone modifier gene mutations in peripheral T-cell lymphoma not otherwise specified. Haematologica. 2018;103(4):679-87.

32. Kim SJ, Kim JH, Ki CS, Ko YH, Kim JS, Kim WS. Epstein-Barr virus reactivation in extranodal natural killer/T-cell lymphoma patients: a previously unrecognized serious adverse event in a pilot study with romidepsin. Ann Oncol. 2016;27(3):508-13.

33. Vitolo U, Trneny M, Belada D, Burke JM, Carella AM, Chua N, Abrisqueta P, Demeter J, Flinn I, Hong X, et al. Obinutuzumab or rituximab plus cyclophosphamide, doxorubicin, vincristine, and prednisone in previously untreated diffuse large B-cell lymphoma. J Clin Oncol. 2017;35(31):3529-37.

34. Zhang W, Su L, Liu L, Gao Y, Wang Q, Su H, Song Y, Zhang H, Shen J, Jing $\mathrm{H}$, et al. Combination of chidamide with the $\mathrm{CHOEP}$ regimen in previously untreated patients with Peripheral T-Cell Lymphoma (PTCL): a prospective, multicenter, Single-Arm, Phase 1b/2 Trial. Blood 2019, 134(Supplement_1):4036.
35. A predictive model for aggressive non-Hodgkin's lymphoma. N Engl J Med. 1993, 329(14):987-94.

36. Cheson BD, Fisher RI, Barrington SF, Cavalli F, Schwartz LH, Zucca E, Lister TA. Recommendations for initial evaluation, staging, and response assessment of Hodgkin and non-Hodgkin lymphoma: the Lugano classification. J Clin Oncol. 2014;32(27):3059-68.

37. Simon R. Optimal two-stage designs for phase II clinical trials. Control Clin Trials. 1989;10(1):1-10.

38. Xu PP, Zhong HJ, Huang YH, Gao XD, Zhao X, Shen Y, Cheng S, Huang $J Y$, Chen SJ, Wang L, et al. B-cell function gene mutations in diffuse large b-cell lymphoma: a retrospective cohort study. EBioMedicine. 2017;16:106-14.

\section{Publisher's Note}

Springer Nature remains neutral with regard to jurisdictional claims in published maps and institutional affiliations.
Ready to submit your research? Choose BMC and benefit from:

- fast, convenient online submission

- thorough peer review by experienced researchers in your field

- rapid publication on acceptance

- support for research data, including large and complex data types

- gold Open Access which fosters wider collaboration and increased citations

- maximum visibility for your research: over 100M website views per year

At BMC, research is always in progress.

Learn more biomedcentral.com/submissions 